\title{
ORIGINAL
}

\section{TENDENCIA, ESTACIONALIDAD Y DISTRIBUCIÓN GEOGRÁFICA \\ DE LA INCIDENCIA DE FRACTURA DE CADERA EN UN ÁREA DE SALUD DE LA COMUNIDAD VALENCIANA (1994-2000)}

\section{José María Tenías (1) y Damian Mifsut Miedes (2)}

(1) Servicio de Medicina Preventiva. Hospital Lluís Alcanyís de Xàtiva

(2) Servicio de Traumatología. Hospital Lluís Alcanyís de Xàtiva

\section{RESUMEN}

Fundamento: La incidencia de fractura de cadera ha sido estimada en nuestro país en periodos cortos de tiempo, sin valorar los cambios temporales de tendencia y estacionalidad. El objetivo de este trabajo es estimar la incidencia de fractura de cadera en un Área de Salud de la Comunidad Valenciana durante 7 años (1994-2000), explorando su tendencia, estacionalidad y los cambios geográficos por zonas de salud y tipo de núcleo, rural o urbano.

Métodos: Estudio descriptivo de los casos de FC en mayores de 45 años. Se excluyeron los pacientes no residentes en el Área, las fracturas patológicas y las producidas por accidente de tráfico. Los cambios temporales y geográficos de la incidencia se estimaron por regresión de Poisson.

Resultados: La incidencia global de fractura de cadera en mayores de 45 años fue de 274 casos por 100000 habitantes y año (IC95\% 259-288). En hombres fue de 149 y en mujeres de 383 casos por 100000 habitantes-año. La distribución temporal mostró una tendencia positiva aunque no significativa, con un incremento medio mensual de la incidencia del $0,04 \%$. Esta tendencia fue mayor para hombres que para mujeres. Se observó una clara estacionalidad, con incidencias relativas menores en los meses de primavera y verano. La incidencia no varió significativamente por zonas de salud ni por el tipo de núcleo urbano o rural.

Conclusiones: La incidencia de FC es similar aunque algo superior a la de otras provincias españolas. Se observa una discreta tendencia positiva y una clara estacionalidad, sin cambios geográficos significativos.

Palabras clave: Fractura de cadera, epidemiología, incidencia

Correspondencia:

José María Tenías Burillo

Servicio de Medicina Preventiva

Hospital Lluís Alcanyís

Crtra. Xátiva-Silla Km 2

46800 Xátiva

Correo electrónico: Tenias_jma@gva.es

\section{ABSTRACT}

Hip Fracture Incidence: Trend, Seasonality and Geographic Distribution in a Health District in the Autonomous

Community of Valencia, Spain (1994-2000)

Background: Hip fracture incidence has been estimated in our country over short time periods without evaluating the changes in trend and seasonality over the course of time. This study is aimed at estimating hip fracture incidence in a Health District in the Autonomous Community of Valencia throughout a seven-year period (1994-2000), delving into its trend, seasonality and the geographical changes by health districts and whether a rural or urban population is involved.

Methods: Descriptive study of the cases of HF among individuals over 45 years of age. The patients not residing within the District, the pathological fractures and those due to traffic accident were not included. The time-related and geographical changes in the incidence were estimated by Poisson regression.

Results: The overall incidence of hip fractures among individuals over 45 years of age was 274 cases/100000 inhabitants/year (CI 95\% 259-288). The incidence among males was 149 cases/100,000 inhabitants/year; being 383 cases/100,000 inhabitants/ year for females. The time-related distribution show a positive, although non-significant trend, with an average $0.04 \%$ monthly increase in the incidence. This trend was greater among males than among females. A clear seasonality, with relatively lower incidences during the spring and summer months, were found to exist. The incidences did not vary significantly among health districts nor by the type of urban or rural population involved.

Conclusions: HF incidence is similar to although somewhat higher than that of other Spanish provinces. A discreet positive trend and a clear seasonality were found, without any significant geographical changes.

Key words: Hip fracture. Epidemiology. Incidence 


\section{INTRODUCCIÓN}

La fractura de cadera (FC) es una complicación de la osteoporosis que conlleva un incremento importante en el coste económico derivado de su atención medica directa ${ }^{1}$. La incidencia de fractura de cadera ha sido estimada en diferentes provincias españolas $^{2-12}$ variando desde 137,9 a 264,7 casos anuales por 100.000 habitantes mayores de 50 años. Sin embargo, los periodos de análisis han sido cortos, lo cual ha dificultado explorar los cambios temporales de la incidencia, tanto en su tendencia como estacionalidad. Solamente en un estudio realizado en la ciudad de Oviedo se ha analizado un periodo amplio de seis años ${ }^{13}$ : Sin embargo, el estudio se llevó a cabo en una cohorte reducida de habitantes sin el objetivo de explorar la evolución temporal en la incidencia de fracturas.

En un estudio reciente se analiza para toda España la incidencia de fractura de cadera en personas mayores de 64 años en un periodo de cuatro años, estimando las variaciones por Comunidades Autónomas ${ }^{14}$. También estudian los porcentajes de casos por estaciones encontrando pocas variaciones, aunque se observa un ligero aumento en otoño e invierno.

Fuera de nuestro país diversos estudios han analizado las variaciones estacionales de este problema de salud, encontrando generalmente incidencias mayores en los meses más fríos del año. Estas diferencias estacionales han intentando ser explicadas por los cambios en las condiciones climatológicas, con resultados desiguales ${ }^{15-19}$.

También se han estudiado las posibles variaciones geográficas en la incidencia de la FC, comparándose en los núcleos urbanos respecto a los rurales ${ }^{20}$.

El objetivo de este estudio es estimar la incidencia global de FC para un periodo de 7 años (1994-2000), su evolución temporal y los cambios geográficos (por zonas de salud y por núcleos rurales o urbanos) en el Área de salud número 13 de la Comunidad Valenciana.

\section{MATERIAL Y MÉTODOS}

Se trata de un estudio observacional, descriptivo en el que se recogieron todos los casos ingresados por fractura osteoporótica de cadera en los dos hospitales de referencia del Área de Salud número 13 de la Comunidad Valenciana (Censo 1996: 180.995 habitantes) durante el período de enero 1994 a diciembre de 2000.

La definición de caso se basó en el registro hospitalario del Conjunto Mínimo Básico de Datos (CMBD). Se seleccionó a aquellos pacientes con un diagnóstico principal con los códigos CIE 820.00 al 820.9.

Se descartaron aquellos casos residentes fuera del área de salud número 13, a las personas menores de 45 años y a aquéllas con una fractura patológica, no osteoporótica (enfermedad de Paget, insuficiencia renal crónica, patología tiroidea) o las producidas a consecuencia de un accidente de tráfico.

Análisis estadístico. Se estimó la incidencia como casos anuales por 100.000 habitantes. Se calculó la incidencia global, por sexo y por grupos de edad. Tomando como base la incidencia en el grupo de menor edad se calcularon los riesgos relativos (RR) para el resto de estratos etarios. La base poblacional se estimó utilizando dos puntos censales (1991 y 2001) y el padrón municipal de 1996, calculando mediante interpolación lineal los habitantes para cada mes y año del periodo de análisis. Para favorecer la comparación con otros estudios la incidencia global se calculó en mayores de 45 años. Por ser previsible una muy baja incidencia entre los 45 y 65 años se analizó separadamente la incidencia global para mayores de 65 años. 
La evolución temporal se estudió analizando la tendencia y estacionalidad de la serie mensual de incidencias de FC desde enero de 1994 a diciembre de 2000. En un modelo de regresión de Poisson se introdujeron dos variables, una representando la tendencia mensual (número de meses transcurridos desde el inicio de la serie) y otra categórica con los meses del año (referencia mes de enero) representando el componente de estacionalidad. Posteriormente, si las estimaciones por meses lo permitían, la estacionalidad se redujo en una variable de 4 categorías que representaban las estaciones climatológicas del año.

La distribución geográfica por sexo y edad se analizó estimando la incidencia en las 17 zonas de salud del área. Finalmente, se estimaron y compararon las incidencias de FC entre los núcleos urbanos (poblaciones con más de 10000 habitantes) y los rurales.

\section{RESULTADOS}

Durante el periodo de análisis (19942000) ingresaron 1.450 pacientes con diag- nóstico de fractura de cadera. 129 fueron excluidos: 58 por ser menores de 45 años, 37 por residir fuera del área, 10 por presentar una fractura patológica no osteoporótica (1 por enfermedad de Paget, 1 por patología tiroidea y 8 por insuficiencia renal crónica) y 53 por haberse producido a consecuencia de un accidente de tráfico.

Las 1.321 fracturas restantes fueron consideradas como FC. En la tabla 1 se resumen las características por edad, sexo, tipo de fractura y estancia hospitalaria.

La incidencia global de $\mathrm{FC}$ en personas mayores de 45 años fue de 274 casos por 100.000 habitantes y año (IC95\% 259-288). En hombres la incidencia fue de 149 casos por $100.000 \mathrm{~h}$ (IC95\% 133-165) y en mujeres de 383 casos por 100000 (IC95\% 359407). La incidencia se incrementó con la edad, tanto en hombres como en mujeres (figura 1). El 87\% de las FC en hombres y el $95 \%$ en mujeres afectaron a pacientes de más de 65 años. La incidencia de FC en este grupo de edad fue muy superior tanto en hombres (326 casos por 100000 habitantes y

Tabla 1

Descripción de los episodios de fractura osteoporótica de cadera en el Área 13 de la Comunidad Valenciana (1994-2000)

\begin{tabular}{|c|c|}
\hline & \multicolumn{1}{|c|}{$\mathbf{N}(\%)$} \\
\hline Edad (años)* & $81(12)$ \\
\hline Sexo & \\
Hombres & $337(25,5 \%)$ \\
Mujeres & $984(74,5 \%)$ \\
Tipo de fractura & \\
Cervical & \\
Pertrocantérea & $515(39,0 \%)$ \\
Localización no especificada & $666(50,4 \%)$ \\
\hline Estancia (días) & $140(10,6 \%)$ \\
\hline
\end{tabular}

* Mediana (intervalo intercuartílico) 
Figura 1

Tasas de incidencia de fractura osteoporótica de cadera por grupos de edad en hombres y mujeres mayores de 45 años. Area de Salud no 13 de la Comunidad Valenciana. Periodo 1994-2000

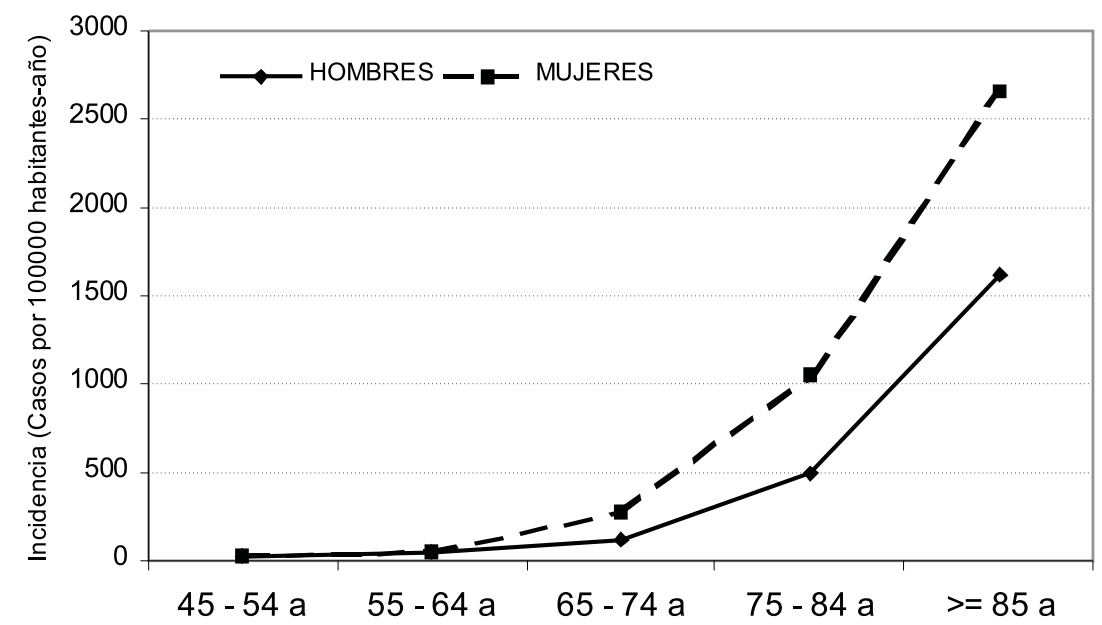

Tabla 2

Evolución temporal de la incidencia de fractura osteoporótica de cadera en el Área 13 de la Comunidad Valenciana. Periodo: 1994-2000

\begin{tabular}{|c|c|}
\hline & RR (IC 95\%)* \\
\hline Tendencia mensual & $1,0004(0,998-1,003)$ \\
\hline Estacionalidad & 1 \\
Invierno (ref.) & $0,837(0,717-0,978)$ \\
Verimavera & $0,843(0,722-0,984)$ \\
Otoño & $1,062(0,917-1,230)$ \\
\hline
\end{tabular}

* Incidencias relativas estimadas por regresión de Poisson

año) como en mujeres (794 casos por 100000 habitantes y año).

La distribución temporal mostró una tendencia positiva aunque no significativa, con un incremento medio mensual de la incidencia de FC de $0,04 \%$ (IC95\%-0,2\% a 0,3\%).
Se observa una clara estacionalidad, con incidencias relativas menores en los meses de primavera y verano respecto al otoño e invierno (tabla 2).

En los hombres, la tendencia en la incidencia de FC fue algo superior (incremento 
Figura 2

Distribución por cuartiles de la incidencia de la fractura osteoporótica de cadera por zonas de Salud en el Área de Salud $\mathbf{n}^{0} 13$ de la Comunidad Valenciana. Los tonos representan la distribución desde el cuartil 1 (Q1) al cuartil 4 (Q4)

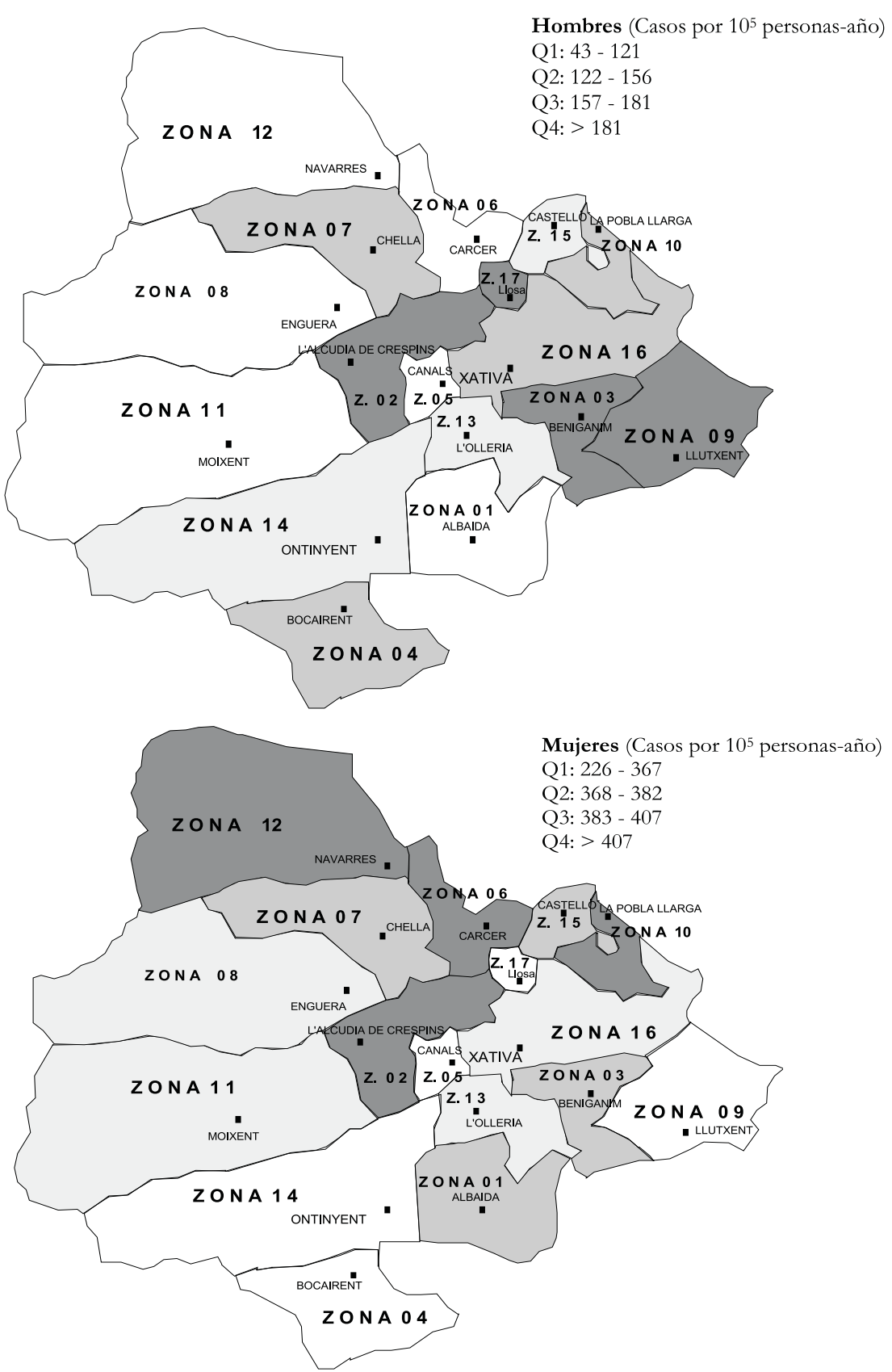


medio mensual del $0,1 \%$ IC95\% $-0,3 \%$ a $0,6 \%)$ que en las mujeres $(0,02 \%$; IC $95 \%$ : $0,2 \%$ a $0,3 \%$ ). La estacionalidad fue similar en ambos sexos, con incidencias mayores en los meses de otoño e invierno.

La incidencia no varió de forma significativa según la zona de salud (figura 2) y tampoco en relación a la residencia urbana o rural del afectado.

\section{DISCUSIÓN}

En este trabajo hemos podido estimar la incidencia de fractura de cadera en un área de salud para un periodo amplio de tiempo. Las cifras globales de incidencia son algo superiores a las de otros trabajos realizados en nuestro país ${ }^{2-12}$. Estas diferencias pueden ser debidas a que nuestro estudio cubre un periodo de tiempo más reciente en el que se ha podido producir un incremento en el número de casos observados de FC.

Las diferencias en la incidencia de FC respecto al género y la edad son fenómenos que consistentemente se observan en la gran mayoría de estudios y se deben principalmente a la mayor frecuencia de osteoporosis en mujeres y en personas de mayor edad. La osteoporosis es la principal causa de FC y en este estudio hemos intentando seleccionar las supuestamente producidas por esta enfermedad. Para ello hemos excluido además de las sufridas por menores de 45 años, las FC por un mecanismo traumático importante (accidentes de tráfico) o las debidas a una enfermedad de base diferente a la osteoporosis (fracturas patológicas). Sin embargo no podemos descartar otras fracturas traumáticas no osteoporóticas debidas a accidentes ocurridos en el domicilio del paciente.

Los cambios temporales en la incidencia de FC no muestran la misma consistencia. Este es el primer estudio que explora simultáneamente la tendencia y estacionalidad de este problema de salud en España.
La tendencia de la serie analizada muestra que la incidencia se ha incrementado en la medida de lo esperado. Aunque el número de casos ha sufrido un incremento importante durante el periodo de análisis (incremento medio anual del 1,6\%) la incidencia de FC en mayores de 45 años se ha mantenido prácticamente constante en nuestro Área. Para las mujeres el incremento de casos observados (incremento medio anual del 1,3\%) es muy similar al esperado por los cambios demográficos, por lo que la tendencia en la incidencia de FC es prácticamente nula. Sin embargo, para los hombres el incremento de casos de FC ha sido mayor que en las mujeres (incremento medio anual del 2,8\%) y superior al incremento de la base poblacional, lo que explica una tendencia positiva. La razón de este incremento mayor en la incidencia de la FC en hombres necesitaría de una evaluación más profunda, aunque en parte se debería a los cambios demográficos observados en nuestra área: entre 1991 y 2001 el número de varones mayores de 65 años se ha incrementado en un $29,1 \%$ y el de mujeres en un $24,4 \%$.

La estacionalidad muestra que la incidencia de FC es mayor en las épocas del año más frías y húmedas. Este hallazgo concuerda con el de otros trabajos ${ }^{(15-19)}$. Se han dados diversas explicaciones a estos cambios estacionales. En un país de climatología similar a la nuestra como Italia se ha explorado este fenómeno comprobándose que la incidencia de fractura de cadera mostraba un movimiento cíclico paralelo a la evolución de algunos indicadores meteorológicos como las precipitaciones en la zona ${ }^{21}$. La FC se produce en una gran mayoría de casos como producto de una caída, tal y como muestran diferentes estudios, algunos de ellos realizados en España ${ }^{11}$. Este tipo de accidente vendría favorecido por unas malas condiciones climatológicas. Por otro lado, en las épocas más frías se produce un descenso marcado de la radiación ultravioleta lo cual dificultaría el metabolismo de la vitamina $\mathrm{D}$ y la absorción del calcio. Este último mecanismo 
sería menos plausible, al menos en una relación a corto plazo.

Los cambios geográficos en la incidencia por zonas de salud no son estadísticamente significativos y tampoco se observan diferencias importantes entre los núcleos urbanos y rurales. La mayor concentración de centros geriátricos en los centros urbanos podría ser la causa de estas diferencias. Algunos trabajos demuestran que a pesar de encontrar diferencias entre núcleos urbanos y rurales éstas no se debían a una desigual disponibilidad de plazas geriátricas ${ }^{20}$. En nuestro estudio no hemos encontrado diferencias entre los dos tipos de población. En nuestra área los centros urbanos son relativamente pequeños, no superando los 30.000 habitantes. Tampoco existe un gran desarrollo de residencias geriátricas, manteniéndose en la gran mayoría de casos en los domicilios familiares con asistencia ambulatoria o a través de la Unidad de Hospitalización Domiciliaria.

Concluyendo, en nuestra área la incidencia de FC es ligeramente superior a la esperada, con diferencias importantes por sexo. La evolución temporal muestra un aumento en el número de casos pero con una tendencia relativamente estable a lo largo de los años. La estacionalidad es importante con picos de incidencia en las estaciones frías y húmedas. En estudios posteriores debería analizarse la influencia de las condiciones meteorológicas en esta distribución estacional. La distribución geográfica es homogénea no observándose variaciones importantes por zonas de salud ni por núcleos urbanos o rurales.

\section{BIBLIOGRAFÍA}

1. Melton LJ 3rd, Gabriel SE, Crowson CS, Tosteson AN, Johnell O, Kanis JA. Cost-equivalence of different osteoporotic fractures. Osteoporos Int. 2003; 14: 383-8.

2. Elffors I, Allander E, Kanis JA, Gullberg B, Johnell $\mathrm{O}$, Dequeker J, et al. The variable incidence of hip fracture in southern Europe: the MEDOS Study. Osteoporos Int 1994; 4: 253-63.

3. Altadill Arregui A, Gómez Alonso C, Virgos Soriano MJ, Díaz Lopez B, Cannata Andia JB. Epidemiologia de la fractura de cadera en Asturias. Med Clin (Barc) 1995; 105: 281-6.

4. Candau E, de la Fuente B, Pozo A, Álvarez JI, Nieto C. Epidemiología de las fracturas de cadera en la provincia de Valladolid en 1991. Rev Esp Enf Met Os 1993; 2: 73-4.

5. Olmos JM, Martínez J, García J, Matorras P, Moreno JJ, Gónzalez-Macias J. Incidencia de la fractura de cadera en Cantabria. Med Clin 1992; 99; 72931 .

6. Rey L, Torrijos A, Armenteros J, Esinoisa A, Munuera L, Gijón J. Fracturas de cadera en el Área V (Madrid). Rev Esp Reumatol 1995; 22: 39-42.

7. Hernández JS, Fernández L, Devesa F, De No L, Ceinos M. Incidencia y costes económicos de las fracturas de fémur asociadas a osteoporosis en Salamanca. Rev Esp Enf Os Metab Min 1992;1 (suppl A): 37.

8. González Domínguez J, Martínez Concha D, Caracuel MA, González Pérez I, Ginés Martínez F, Gala $\mathrm{M}$, et al. Estudio epidemiológico de las fracturas de cadera de la provincia de Córdoba. Rev Esp Reumatol 1995; 22: 10-14.

9. Sosa M, Segarra MC, Hernández D, González A Liminana JM, Betancor P. Epidemiology of proximal femoral fracture in Gran Canaria (Canary Islands). Age Ageing 1993;22: 285-8.

10. Díez A, Puig J, Martinez MT, Díez JL, Aubia J, Vivancos J. Epidemiology of fractures of the proximal femur associated with osteoporosis in Barcelona, Spain. Calcif Tissue Int. 1989 Jun;44:382-6.

11. Arboleya LR, Castro MA, Bartolomé E, Gervas L, Vega R. Epidemiologia de la fractura osteoporótica de cadera en la provincia de Palencia. Rev Clin Esp. 1997; 197:611-7.

12. Izquierdo Sánchez M, Ochoa Sangrador C, Sánchez Blanco I, Hidalgo Prieto MC, Lozano del Valle F, Martín González T. Epidemiología de la fractura osteoporótica de cadera en la provincia de Zamora (1993). Rev Esp Salud Publica 1997; 71: 357-67.

13. Naves Díaz M, Díaz López B, Gómez Alonso C, Altadill Arregui A, Rodríguez Rebollar A, Cannata 
Andía JB. Estudio de incidencia de fracturas osteoporóticas en una cohorte mayor de 50 años durante un periodo de 6 años de seguimiento. Med Clin (Barc) 2000; 115: 650-3

14. Serra JA, Garrido G, Vidán M, Marañón E, Brañas F, Ortiz J. Epidemiologia de la fractura de cadera en ancianos en España. An Med Interna 2002; 19: 389-95.

15. Levy AR, Bensimon DR, Mayo NE, Leighton HG. Inclement weather and the risk of hip fracture. Epidemiology 1998; 9: 172-77.

16. Chiu KY, Ng TP, Chow SP. Seasonal variation of fractures of the hip in elderly people. Injury 1996; 27: 333-6.

17. Lau EM, Gillespie BG, Valenti L, O’Connell D. The seasonality of hip fracture and its relationship with weather conditions in New South Walles. Aust J Public Helath 1995; 19: 76-80.
18. Jacobsen SJ, Sargent DJ, Atkinson EJ, O'Fallon WM, Melton LJ 3rd. Population-based study of the contribution of weather to hip fracture seasonality. Am J Epidemiol 1995; 141: 79-83.

19. Lofthus CM, Osnes EK, Falch JA, Kaastad TS, Kristiansen IS, Nordsletten L, et al. Epidemiology of hip fractures in Oslo, Norway. Bone 2001; 29: 413-8.

20. Chevalley T, Herrmann FR, Delmi M, Stern R, Hoffmeyer P, Rapin $\mathrm{CH}$, et al. Evaluation of the age-adjusted incidence of hip fractures between urban and rural areas: the difference is not related to the prevalence of institutions for the elderly. Osteoporos Int 2002; 13:113-8.

21. Cannigia M, Morreale P. Epidemiology of hip fractures in Siena, Italy, 1975-1985. Clin Orthop 1989; 238: 131-8. 